\title{
Classical and quantum Lemaître-Tolman-Bondi model for the nonmarginal case
}

\author{
Claus Kiefer* \\ Institut für Theoretische Physik, Universität zu Köln, Zülpicher Strasse 77, 50937 Köln, Germany, \\ and Max-Planck-Institut für Gravitationsphysik, Am Mühlenberg 1, 14476 Golm, Germany \\ Jakob Müller-Hill ${ }^{\dagger}$ \\ Institut für Theoretische Physik, Universität zu Köln, Zülpicher Strasse 77, 50937 Köln, Germany \\ Cenalo Vaz \\ Department of Physics, University of Cincinnati, Cincinnati, Ohio 45221-0011, USA
}

(Received 8 December 2005; published 22 February 2006)

\begin{abstract}
We extend the classical and quantum treatment of the Lemaitre-Tolman-Bondi (LTB) model to the nonmarginal case (defined by the fact that the shells of the dust cloud start with a nonvanishing velocity at infinity). We present the classical canonical formalism and address with particular care the boundary terms in the action. We give the general relation between dust time and Killing time. Employing a lattice regularization, we then derive and discuss for particular factor orderings exact solutions to all quantum constraints.
\end{abstract}

DOI: 10.1103/PhysRevD.73.044025

PACS numbers: 04.60.Ds, 04.70.Dy

\section{INTRODUCTION}

As long as a full quantum theory of gravity is not available, it is important to address the quantization of particular models. Among the most interesting ones are systems of spherically symmetric gravity plus matter, because they are sufficiently nontrivial and nevertheless simple enough for some technical treatment. Moreover, they find physical applications in the study of gravitational collapse and quantum aspects of black holes.

In this paper we discuss the Lemaitre-Tolman-Bondi (LTB) model which describes a self-gravitating dust cloud. It has originally been introduced by Lemaître [1] in order to study cosmology where it has indeed found interesting applications, cf. [2] for details and references. Here the focus is on the canonical (Hamiltonian) formalism for both the classical and the quantum LTB model. The classical part is mainly intended as a preparation for the quantum model but it exhibits interesting aspects on its own.

The physical questions that one eventually seeks to address include the issue of singularity avoidance in the quantum theory, the quantum evolution of black holes, and the role of the naked classical singularities in quantum gravity. In the simpler case of a single dust shell, for example, it was shown by an explicit construction that a unitary quantum theory exists in which the classical blackhole singularity is avoided, cf. [3,4]. In fact, a wave packet describing a classical shell evolves into a superposition of collapsing and expanding shell and yields destructive interference at the place of the classical singularity. The formalism of loop quantum gravity has also been applied

\footnotetext{
*Electronic address: kiefer@thp.uni-koeln.de

${ }^{\dagger}$ Electronic address: jmh@thp.uni-koeln.de

*Electronic address: vaz@physics.uc.edu
}

to spherically symmetric systems and it has been claimed that the singularities are avoided [5].

In recent years, earlier works on the canonical quantization of the Schwarzschild black hole [6,7] have been applied to develop a canonical description of the collapse of a marginally bound, spherical, timelike dust cloud [8,9]. "Marginally bound" means that the various shells of the dust cloud start with vanishing velocity at infinity. Quantization of this classical dust system leads to the Wheeler-DeWitt (WDW) equation for the wave functional describing the quantum collapse. As special cases, it was possible to obtain the Bekenstein mass spectrum and statistical entropy of the charged and uncharged black hole [10]. Later, in [11], it was shown that the semiclassical (WKB) treatment of the Schwarzschild black hole in this canonical picture describes Hawking's thermal radiation and in [12], going beyond the WKB approximation, that quantum corrections render the spectrum of the radiation nonthermal.

Here we again apply quantum geometrodynamics and the Wheeler-DeWitt equation to the LTB model in order to see how far this approach can be developed. In contrast to the earlier works, we consider the generic case, that is, the case including the nonmarginal models for which the classical shells start with a nonvanishing velocity at infinity. This paper is organized as follows: in Sec. II we introduce the LTB model and present the canonical formalism for the general models. Some technical details are relegated to Appendix A. In Sec. III we perform a lattice regularization and find for a particular set of factor orderings exact solutions to both the Wheeler-DeWitt equation and the diffeomorphism constraint. The uniqueness of this set of solutions for the given ansatz is shown in Appendix B. Section IV presents a brief summary and an outlook on possible future work. 


\section{THE CLASSICAL LTB MODEL}

\section{A. Metric and classical solutions}

The LTB model describes a self-gravitating dust cloud. Its energy-momentum tensor reads $T_{\mu \nu}=\epsilon(\tau, \rho) u_{\mu} u_{\nu}$, where $u^{\mu}=u^{\mu}(\tau, \rho)$ is the four-velocity vector of a dust particle with proper time $\tau$ and labeled by $\rho$ ( $\rho$ thus labels the various shells that together form the dust cloud). The line element for the LTB spacetime is given by

$$
\begin{aligned}
d s^{2}= & -d \tau^{2}+\frac{\left(\partial_{\rho} R\right)^{2}}{1+2 E(\rho)} d \rho^{2} \\
& +R^{2}(\rho)\left(d \theta^{2}+\sin ^{2} \theta d \phi^{2}\right) .
\end{aligned}
$$

Inserting this expression into the Einstein equations leads to

$$
\epsilon(\tau, \rho)=\frac{\partial_{\rho} F}{R^{2} \partial_{\rho} R} \quad \text { and } \quad\left(\partial_{\tau} R\right)^{2}=\frac{G F}{R}+2 E,
$$

where $F(\rho)$ is some non-negative function, $G$ is the gravitational constant, which we set equal to one in the following (except for some key expressions where it is retained), and we set $c=1$ throughout. The case of collapse is described by $\partial_{\tau} R(\tau, \rho)<0$.

There exists still the freedom to rescale the shell index $\rho$. This can be fixed by demanding

$$
R(0, \rho)=\rho,
$$

so that for $\tau=0$ the label coordinate $\rho$ is equal to the curvature radius $R$. Now we can express the functions $F(\rho)$ and $E(\rho)$ in terms of the energy density $\epsilon$ at $\tau=0$. From (2) one gets

$$
\begin{gathered}
F(\rho)=\int_{0}^{\rho} \epsilon(0, \tilde{\rho}) \tilde{\rho}^{2} d \tilde{\rho} \\
E(\rho)=\left[\partial_{\tau} R(\tau=0, \rho)\right]^{2}-\frac{1}{\rho} \int_{0}^{\rho} \epsilon(0, \tilde{\rho}) \tilde{\rho}^{2} d \tilde{\rho} .
\end{gathered}
$$

The interpretation of these quantities is that $F(\rho) / 2$ is the active gravitating mass inside of $R(\tau, \rho)$, while $E(\rho) / 2$ is the total energy of the shell labeled by $\rho$. The marginally bound models are defined by $E(\rho)=0$. In the present paper we discuss the general case which includes the nonmarginal case defined by $E(\rho) \neq 0$.

The solution of (2) is given by

$$
\tau-\tau_{0}(\rho)=-\frac{R^{3 / 2}(\tau, \rho) Q\left(-\frac{2 E(\rho) R(\tau, \rho)}{F(\rho)}\right)}{\sqrt{F(\rho)}},
$$

where $\tau_{0}$ is a constant of integration that can be fixed by (3) to read

$$
\tau_{0}(\rho)=\frac{\rho^{3 / 2} Q\left(-\frac{2 E \rho}{F}\right)}{\sqrt{F}} .
$$

The function $Q(y)$ is defined by the expressions

$$
\begin{gathered}
Q(y)=\left(\frac{\arcsin (\sqrt{y})}{y^{3 / 2}}-\frac{\sqrt{1-y}}{y}\right) \text { for } 1 \geq y>0 \\
Q(y)=\frac{2}{3} \text { for } y=0 \\
Q(y)=\left(\frac{\operatorname{arcsinh}(\sqrt{-y})}{(-y)^{3 / 2}}-\frac{\sqrt{1-y}}{y}\right) \text { for } 0>y \geq-\infty,
\end{gathered}
$$

where $Q>0$ and $1 \geq y \geq-\infty$.

Equation (6) shows that at the dust proper time $\tau=$ $\tau_{0}(\rho)$ the shell labeled by $\rho$ has reached a curvature radius $R=0$, that is, the physical singularity. So $\tau$ can only take values between $-\infty$ and $\tau_{0}(\rho)$.

\section{B. Hamiltonian formalism}

For the canonical formalism one starts with the general ansatz for a spherically symmetric line element,

$$
d s^{2}=-N^{2} d t^{2}+L^{2}\left(d r-N^{r} d t\right)^{2}+R^{2} d \Omega^{2},
$$

where $N$ and $N^{r}$ are the lapse and shift function, respectively. The canonical momenta are given by

$$
\begin{gathered}
P_{L}=\frac{R}{N}\left(-\dot{R}+N^{r} R^{\prime}\right), \\
P_{R}=\frac{1}{N}\left[-L \dot{R}-\dot{L} R+\left(N^{r} L R\right)^{\prime}\right] .
\end{gathered}
$$

A dot denotes a derivative with respect to coordinate time $t$, while a prime denotes a derivative with respect to $r$. All variables are functions of $t$ and $r$.

A Legendre transformation from the Einstein-Hilbert action leads to

$$
S_{\mathrm{EH}}=\int d t \int_{0}^{\infty} d r\left(P_{L} \dot{L}+P_{R} \dot{R}-N H^{g}-N^{r} H_{r}^{g}\right)+S_{\partial \Sigma},
$$

where the Hamiltonian and the diffeomorphism (momentum) constraint are given by

$$
\begin{gathered}
H^{g}=-G\left(\frac{P_{L} P_{R}}{R}-\frac{L P_{L}^{2}}{2 R^{2}}\right)+\frac{1}{G}\left[-\frac{L}{2}-\frac{R^{\prime 2}}{2 L}+\left(\frac{R R^{\prime}}{L}\right)^{\prime}\right], \\
H_{r}^{g}=R^{\prime} P_{R}-L P_{L}^{\prime},
\end{gathered}
$$

respectively, and the boundary action $S_{\partial \Sigma}$ is discussed below.

The total action is the sum of (14) and an action $S^{d}$ describing the dust. The canonical formalism for the latter was developed in [13], cf. also [9]. It reads

$$
S^{d}=\int d t \int_{0}^{\infty} d r\left(P_{\tau} \dot{\tau}-N H^{d}-N^{r} H_{r}^{d}\right),
$$

where the Hamiltonian and momentum constraints are 


$$
H^{d}=P_{\tau} \sqrt{1+\frac{\tau^{\prime 2}}{L^{2}}} \text { and } H_{r}^{d}=\tau^{\prime} P_{\tau} .
$$

In principle one would prefer to take a fundamental field (e.g. a scalar field) for the matter part. However, this would make the formalism much less tractable [14]. Moreover, the main features discussed here already exhibit themselves for the dust model.

\section{Mass function in terms of canonical variables}

In the following we shall write the mass function $F(\rho)$, which was introduced in (4), from the canonical data. This is essential for deriving consistent falloff conditions that are appropriate for a realistic collapse model. For the Schwarzschild case this was done in [7], while for the marginal solutions $(E=0)$ this was done in [9]. Here we shall extend the derivation to the nonmarginal case.

We start by demanding that the spacetime described by the metric (11) be embedded in a LTB spacetime. Considering the LTB metric (1), a foliation described by functions $\tau(r, t)$ and $\rho(r, t)$ leads to

$$
\begin{aligned}
d s^{2}= & -\left(\dot{\tau}^{2}-\frac{\dot{\rho}\left(\partial_{\rho} R\right)^{2}}{1+2 E}\right) d t^{2}-\left(2 \dot{\tau} \tau^{\prime}-\frac{2 \dot{\rho} \rho^{\prime}\left(\partial_{\rho} R\right)^{2}}{1+2 E}\right) d t d r \\
& +\left(\frac{\rho^{\prime 2}\left(\partial_{\rho} R\right)^{2}}{1+2 E}-\tau^{\prime 2}\right) d r^{2}+R^{2} d \Omega
\end{aligned}
$$

Comparison with (11) gives the following three equations:

$$
\begin{gathered}
N^{2}-L^{2} N^{r 2}=\dot{\tau}^{2}-\frac{\dot{\rho}^{2}\left(\partial_{\rho} R\right)^{2}}{1+2 E}, \\
L^{2} N^{r}=\frac{\dot{\rho} \rho^{\prime}\left(\partial_{\rho} R\right)^{2}}{1+2 E}-\dot{\tau} \tau^{\prime}, \\
L^{2}=\frac{\rho^{\prime 2}\left(\partial_{\rho} R\right)^{2}}{1+2 E}-\tau^{\prime 2} .
\end{gathered}
$$

Eliminating $N^{r}$ from (20) with the help of (21) gives

$$
N^{2}=\left(\partial_{\rho} R\right)^{2} \frac{\left(\rho^{\prime} \dot{\tau}-\tau^{\prime} \dot{\rho}\right)^{2}}{L^{2}(1+2 E)} .
$$

Defining $\mathcal{F} \equiv 1-F / R$ we can rewrite Einstein's equation (2) as

$$
\left(\partial_{\tau} R\right)^{2}=2 E+1-\mathcal{F} .
$$

Now we insert the expressions for lapse function (23) and shift vector (21) into the expression for the canonical momentum $P_{L}$ (12). Defining $\bar{R} \equiv\left(\partial_{\rho} R\right) / \sqrt{1+2 E}$ and $J \equiv \rho^{\prime} \dot{\tau}-\dot{\rho} \tau^{\prime}$ we have

$$
\frac{L P_{L}}{R}=\frac{1}{\bar{R}}\left(-\dot{R}+\left(L^{2} \dot{\rho} \rho^{\prime} \bar{R}^{2}-\tau^{\prime} \dot{\tau}\right) R^{\prime}\right) .
$$

Using the expression (22) for $L$ on the right-hand side as well as

$$
\begin{gathered}
\dot{R}=\dot{\tau} \partial_{\tau} R+\dot{\rho} \partial_{\rho} R=\dot{\tau} \partial_{\tau} R+\bar{R} \dot{\rho} \sqrt{1+2 E}, \\
R^{\prime}=\tau^{\prime} \partial_{\tau} R+\rho^{\prime} \partial_{\rho} R=\tau^{\prime} \partial_{\tau} R+\bar{R} \rho^{\prime} \sqrt{1+2 E}
\end{gathered}
$$

we get

$$
\frac{L P_{L}}{R} \bar{R} J=-\bar{R} J \rho^{\prime} \partial_{\tau} R-\bar{R} \sqrt{1+2 E} \tau^{\prime} J,
$$

and therefore

$$
\begin{gathered}
\frac{L P_{L}}{R}=-\frac{\left(R^{\prime}-\tau^{\prime} \partial_{\tau} R\right) \partial_{\tau} R}{\sqrt{1+2 E}}-\sqrt{1+2 E} \tau^{\prime} \\
\stackrel{(2)}{=} \frac{R^{\prime} \sqrt{2 E+1-\overline{\mathcal{F}}}}{\sqrt{1+2 E}}-\frac{\mathcal{F} \tau^{\prime}}{\sqrt{1+2 E}} .
\end{gathered}
$$

Solving this equation for $\tau^{\prime}$ gives

$$
\tau^{\prime}=\frac{1}{\mathcal{F}}\left(R^{\prime} \sqrt{1+2 E-\overline{\mathcal{F}}}-\frac{L P_{L} \sqrt{1+2 E}}{R}\right) .
$$

Inserting this expression into (22) yields

$$
\begin{aligned}
L^{2}= & \bar{R}^{2} \rho^{\prime 2}-\tau^{\prime 2} \\
= & \frac{1}{\sqrt{1+2 E} \mathcal{F}}\left(R^{\prime}(1+2 E)\right. \\
& -\sqrt{1+2 E} \sqrt{\left.1+2 E-\mathcal{F} L P_{L} / R\right)^{2}}-\tau^{\prime 2} \\
= & \frac{R^{\prime 2}}{\mathcal{F}^{2}}-\frac{L^{2} P_{L}^{2}}{R^{2}},
\end{aligned}
$$

which leads to (reinserting $G$ )

$$
\mathcal{F}=\frac{R^{\prime 2}}{L^{2}}-G^{2} \frac{P_{L}^{2}}{R^{2}} .
$$

We can thus express $F$ locally in terms of the canonical data as follows:

$$
F=R\left[1+G^{2} \frac{P_{L}^{2}}{R^{2}}-\frac{R^{\prime 2}}{L^{2}}\right]
$$

This is the same expression as was obtained in [9] for the marginal models. It thus possesses a much wider range of applicability and holds, in fact, for all cases.

Since $R=F$ at the horizon, $\mathcal{F}=0$ there. We can check that though $\mathcal{F}$ appears in the denominator of $(31), \tau^{\prime}$ is well behaved at the horizon, as it should be:

$$
\tau^{\prime} \stackrel{\mathcal{F} \rightarrow 0}{\longrightarrow} \frac{1}{2}\left(R^{\prime}+L\right) .
$$

As in the case of the Schwarzschild black hole [7] and the marginal LTB model [9], one can make a canonical transformation in order to elevate the mass function $F$ to a canonical coordinate. The expressions are the same as in these earlier papers. The canonical transformation is 


$$
\left(\tau, R, L, P_{\tau}, P_{R}, P_{L}\right) \rightarrow\left(\tau, R, F, P_{\tau}, \bar{P}_{R}, P_{F}\right),
$$

where

$$
\bar{P}_{R}=P_{R}-\frac{L P_{L}}{2 R}-\frac{L P_{L}}{2 R \mathcal{F}}-\frac{\Delta}{R L^{2} \mathcal{F}},
$$

with

$$
\Delta=\left(R R^{\prime}\right)\left(L P_{L}\right)^{\prime}-\left(R R^{\prime}\right)^{\prime}\left(L P_{L}\right) .
$$

The action in the new canonical variables then reads

$$
\begin{aligned}
S_{\mathrm{EH}}= & \int d t \int_{0}^{\infty} d r\left(P_{\tau} \dot{\tau}+\bar{P}_{R} \dot{R}+P_{F} \dot{F}-N H-N^{r} H_{r}\right) \\
& +S_{\partial \Sigma},
\end{aligned}
$$

where the new constraints are

$$
\begin{gathered}
H=-\frac{1}{2 L}\left(\frac{F^{\prime} R^{\prime}}{G \mathcal{F}}+4 G \mathcal{F} P_{F} \bar{P}_{R}\right)+P_{\tau} \sqrt{1+\frac{\tau^{\prime}}{L^{2}}}, \\
H_{r}=\tau^{\prime} P_{\tau}+R^{\prime} \bar{P}_{R}+F^{\prime} P_{F} .
\end{gathered}
$$

We shall now discuss the boundary action $S_{\partial \Sigma}$ in more detail.

\section{Boundary action}

Boundary terms are obtained from a careful discussion of the falloff conditions for the canonical variables. This was investigated for the marginal case in [9]. For the nonmarginal case, this derivation remains unchanged if $E \rightarrow 0$ for $r \rightarrow 0$. But this follows if one assumes that the initial density profile is regular at the center: Taking $R(0, r)=r$ (this is just a choice of scaling), then

$$
F(r)=\int r^{2} \mu(r) d r
$$

where $\mu(r)=\epsilon(0, r)$ is some initial density profile, cf. (4). For a profile that is regular at the center, choose

$$
\mu(r)=\sum_{n=0}^{\infty} \mu_{n} r^{n},
$$

which gives

$$
F(r)=\sum_{n=0}^{\infty} F_{n} r^{n+3}
$$

As the center of the collapsing cloud is taken to be at rest in a spherically symmetric collapse, the velocity profile $\partial_{\tau} R(0, r)$, must, to leading order, behave as some positive power of the label coordinate, $r$. It then follows from the "velocity equation" (24) that $E$ behaves as $r^{2}$ or higher. In particular, $E \rightarrow 0$ as $r \rightarrow 0$.

The only boundary term is obtained from the variation with respect to $L$ and reads

$$
\int d t N_{+}(t) \delta M_{+}(t)
$$

where $N_{+}(t) \equiv N(t, r \rightarrow \infty)$ is the lapse function at infinity and $M_{+}(t) \equiv F(r \rightarrow \infty) / 2$ is the Arnowitt-Deser-Misner (ADM) mass. To avoid the conclusion that $N_{+}(t)$ is constraint to vanish, which would freeze the evolution at infinity, the boundary term has to be canceled by an appropriate boundary action. This can be achieved by adding the surface action

$$
S_{\partial \Sigma}=-\int d t N_{+}(t) M_{+}(t) .
$$

Since varying $N_{+}$would lead to zero mass, Kuchař has argued in [7] that $N_{+}$has to be treated as a prescribed function. The lapse function gives the ratio of proper time to coordinate time in the direction normal to the foliation. Since $N^{r}(r)$ vanishes for $r \rightarrow \infty$, the time evolution at infinity is generated along the world lines of observers with $r=$ const. If we introduce the proper time, $\bar{\tau}_{+}$, of these observers as a new variable, we can express the lapse function in the form $N_{+}(t)=\dot{\bar{\tau}}_{+}(t)$. This leads to

$$
S_{\partial \Sigma}=-\int d t M_{+} \dot{\bar{\tau}}_{+} .
$$

Thus we have removed the necessity of fixing the lapse function at infinity. (In [7] this is called "parametrization at infinities.")

In [9], the proper time $\bar{\tau}_{+}$was identified with the dust proper time at infinity, $\tau_{+}$. By realizing that $\bar{\tau}_{+}$is a priori not directly related to $\tau_{+}$, we propose here a different treatment. Extending the treatment from the Schwarzschild case [7] to here, the aim is to cast the homogeneous part of the action into Liouville form and to find a transformation to new canonical variables that absorb the boundary terms. This can be done by introducing the mass density $\Gamma \equiv F^{\prime}$ as a new canonical variable and using that $F(0)=0$ (which is appropriate for a collapse situation). Part of the Liouville form can then be rewritten as follows:

$$
\begin{aligned}
\bar{\theta} \equiv & \int_{0}^{\infty} d r P_{F} \delta F-M_{+} \delta \bar{\tau}_{+} \\
= & \int_{0}^{\infty} d r P_{F}(r) \int_{0}^{r} d r^{\prime} \delta \Gamma\left(r^{\prime}\right)+\bar{\tau}_{+} \delta M_{+}-\delta\left(M_{+} \bar{\tau}_{+}\right) \\
= & \int_{0}^{\infty} d r P_{F}(r) \int_{0}^{r} d r^{\prime} \delta \Gamma\left(r^{\prime}\right)+\frac{\bar{\tau}_{+}}{2} \int_{0}^{\infty} d r^{\prime} \delta \Gamma\left(r^{\prime}\right) \\
& -\delta\left(M_{+} \bar{\tau}_{+}\right) \\
= & \int_{0}^{\infty} d r \delta \Gamma(r) \int_{r}^{\infty} d r^{\prime} P_{F}\left(r^{\prime}\right)+\int_{0}^{\infty} d r^{\prime} \delta \Gamma\left(r^{\prime}\right) \frac{\bar{\tau}_{+}}{2} \\
& -\delta\left(M_{+} \bar{\tau}_{+}\right)
\end{aligned}
$$




$$
=\int_{0}^{\infty} d r \delta \Gamma\left(\frac{\bar{\tau}_{+}}{2}+\int_{r}^{\infty} d r^{\prime} P_{F}\left(r^{\prime}\right)\right)-\delta\left(M_{+} \bar{\tau}_{+}\right) .
$$

In the calculation we have used the identity

$$
\int_{0}^{\infty} d r P_{F}(r) \int_{0}^{r} d r^{\prime} \delta \Gamma\left(r^{\prime}\right)=\int_{0}^{\infty} d r \delta \Gamma(r) \int_{r}^{\infty} d r^{\prime} P_{F}\left(r^{\prime}\right),
$$

which can be seen by integrating

$$
\begin{aligned}
& \left(\int_{\infty}^{r} d r^{\prime} P_{F}\left(r^{\prime}\right) \int_{0}^{r} d r^{\prime} \delta \Gamma\left(r^{\prime}\right)\right)^{\prime} \\
& \quad=P_{F}(r) \int_{0}^{r} d r^{\prime} \delta \Gamma\left(r^{\prime}\right)-\delta \Gamma(r) \int_{r}^{\infty} d r^{\prime} P_{F}\left(r^{\prime}\right)
\end{aligned}
$$

from 0 to $\infty$. The left-hand side gives zero and the rest the desired equation.

From line (54) we can read off that $P_{\Gamma}=\bar{\tau}_{+} / 2+$ $\int_{r}^{\infty} d r P_{F}$. Thus we see that $P_{\Gamma}(\infty)=\bar{\tau}_{+} / 2$. As will become clear from the next subsection, this means that the Killing time at infinity matches the prescribed function $\bar{\tau}_{+}$. Thus the new action reads

$$
S_{\mathrm{EH}}=\int d t \int_{0}^{\infty} d r\left(P_{\tau} \dot{\tau}+\bar{P}_{R} \dot{R}+P_{\Gamma} \dot{\Gamma}-N H^{g}-N^{r} H_{r}^{g}\right) .
$$

The constraints in the new variables are

$$
\begin{gathered}
H=-\frac{1}{2 L}\left(\frac{\Gamma R^{\prime}}{G \mathcal{F}}-4 G \mathcal{F} P_{\Gamma}^{\prime} \bar{P}_{R}\right)+P_{\tau} \sqrt{1+\frac{\tau^{\prime}}{L^{2}}}, \\
H_{r}=\tau^{\prime} P_{\tau}+R^{\prime} \bar{P}_{R}-\Gamma P_{\Gamma}^{\prime} .
\end{gathered}
$$

The Hamiltonian constraint can be greatly simplified if the momentum constraint is used to eliminate $P_{F}$. The details of the calculation can be found in Appendix A. The constraints (58) and (59) can then be replaced by the following equivalent set:

$$
\begin{gathered}
H=G\left(P_{\tau}^{2}+\mathcal{F} \bar{P}_{R}^{2}\right)-\frac{\Gamma^{2}}{4 G \mathcal{F}} \approx 0, \\
H_{r}=\tau^{\prime} P_{\tau}+R^{\prime} \bar{P}_{R}-\Gamma P_{\Gamma}^{\prime} \approx 0 .
\end{gathered}
$$

These equations will be used as the starting point for the quantization in Sec. III.

We emphasize that the relative sign between the dust kinetic term and the gravitational kinetic term can change because $\mathcal{F}>0(<0)$ outside (inside) the horizon. Since this change of sign is already present in (58), it was not introduced by using the momentum constraint to eliminate $P_{F}$. In fact, this change of sign of the gravitational part is also present in the corresponding expression for the Schwarzschild black hole, cf. Eq. (117) in [7]. Thus, it arises due to the choice of the new canonical variables, which are obtained by embedding the spatial spherically symmetric hypersurface into a LTB or Schwarzschild spacetime. To define $F$, we had to use the canonical momentum $P_{L}$. Thus we have mixed the original canonical coordinates with their momenta, which means that the new configuration space, spanned by $\tau, R$, and $F$, is different from the original one containing the three-geometries, which is spanned by $\tau, R$, and $L$. The change of sign is of fundamental interest in the quantum theory because the original WDW equation has a (locally) hyperbolic kinetic term, which is of importance for the formulation of the proper boundary value problem $[4,15]$. A change of sign has hitherto been noticed for the WDW equation in the presence of a nonminimally coupled scalar field [16].

\section{E. Relation between dust proper time and Killing time}

In this subsection we explain under which circumstances it makes sense to interpret $P_{\Gamma}$ as Killing time. Equation (31) is given in the new variables by the expression

$$
\tau^{\prime}=2 P_{\Gamma}^{\prime} \sqrt{1+2 E}+\frac{R^{\prime}}{\mathcal{F}} \sqrt{1+2 E-\mathcal{F}} .
$$

Defining $a \equiv 1 / \sqrt{1+2 E}$ gives

$$
\tau^{\prime}=\frac{2 P_{\Gamma}^{\prime}}{a}+R^{\prime} \frac{\sqrt{1-a^{2} \mathcal{F}}}{a \mathcal{F}} .
$$

If the mass density vanishes for all $r$ greater than a given $r_{b}$, and if $E$ is constant for all $r$ greater than $r_{b}$, Eq. (63) can be integrated. This yields

$$
\begin{aligned}
a \tau= & 2 P_{\Gamma}+\int d R \frac{\sqrt{1-a^{2} \mathcal{F}}}{\mathcal{F}} \\
= & 2 P_{\Gamma}+F\left(\frac{\sqrt{1-a^{2} \mathcal{F}}}{1-\mathcal{F}}+\ln \left|\frac{1-\sqrt{1-a^{2}} \overline{\mathcal{F}}}{1+\sqrt{1-a^{2} \mathcal{F}}}\right|\right. \\
& \left.-\frac{1-a^{2} / 2}{\sqrt{1-a^{2}}} \ln \left|\frac{\sqrt{1-a^{2}} \overline{\mathcal{F}}-\sqrt{1-a^{2}}}{\sqrt{1-a^{2} \overline{\mathcal{F}}}+\sqrt{1-a^{2}}}\right|\right) .
\end{aligned}
$$

The result can also be written in the form

$$
\begin{aligned}
a \tau= & 2 P_{\Gamma}+F\left[\frac{\sqrt{1-a^{2} \mathcal{F}}}{1-\mathcal{F}}-2 \tanh ^{-1} \sqrt{1-a^{2} \mathcal{F}}\right. \\
& \left.+\frac{2-a^{2}}{\sqrt{1-a^{2}}} \tanh ^{-1} \frac{\sqrt{1-a^{2}}}{\sqrt{1-a^{2} \mathcal{F}}}\right] .
\end{aligned}
$$

Here we have assumed that $0<a \leq 1$ (corresponding to $E \geq 0$ ). Equation (24) guarantees that the discriminant $1-$ $a^{2} \mathcal{F}$ is non-negative. This condition may be violated in the quantum theory, so it is of interest to give the result for all cases. For $a>1$ but $R<F a^{2} /\left(a^{2}-1\right)$, the result can be found by analytic continuation to read 


$$
\begin{aligned}
a \tau= & 2 P_{\Gamma}+F\left[\frac{\sqrt{1-a^{2} \mathcal{F}}}{1-\mathcal{F}}+\ln \left|\frac{1-\sqrt{1-a^{2} \mathcal{F}}}{1-\sqrt{1-a^{2} \mathcal{F}}}\right|\right. \\
& \left.+\frac{2-a^{2}}{\sqrt{a^{2}-1}} \tan ^{-1} \frac{\sqrt{a^{2}-1}}{\sqrt{1-a^{2} \mathcal{F}}}\right] .
\end{aligned}
$$

Another analytic continuation then gives the result in the region $R>F a^{2} /\left(a^{2}-1\right)$,

$$
\begin{aligned}
a \tau= & 2 P_{\Gamma}+i F\left[\frac{\sqrt{a^{2} \mathcal{F}-1}}{1-\mathcal{F}}-2 \tan ^{-1} \sqrt{a^{2} \mathcal{F}-1}\right. \\
& \left.+\frac{\left(1-a^{2} / 2\right)}{\sqrt{a^{2}-1}} \ln \left|\frac{\sqrt{a^{2} \mathcal{F}-1}-\sqrt{a^{2}-1}}{\sqrt{a^{2} \mathcal{F}-1}+\sqrt{a^{2}-1}}\right|\right] .
\end{aligned}
$$

These expressions will be of relevance in the quantum theory, see Sec. III.

We know from Birkhoff's theorem that the spacetime around a collapsing dust cloud is given by the Schwarzschild solution. In [7] it was shown for the Schwarzschild geometry that $2 P_{\Gamma}$ is equal to the Killing time $T$. Thus the equation derived above connects the dust proper time with the Killing time at the boundary $r_{b}$. For small $\Gamma$ and $E^{\prime}$ the relationship may still be used, since then we have a small amount of dust propagating in a Schwarzschild background; only in this case the concept of Killing time makes sense. In the limit $a \rightarrow 1(E \rightarrow 0)$ we obtain

$$
\tau=T+2 \sqrt{F}\left[\sqrt{R}-\frac{\sqrt{F}}{2} \ln \left(\frac{\sqrt{R}+\sqrt{F}}{\sqrt{R}-\sqrt{F}}\right)\right],
$$

which is identical to the relation used in [11] for the marginal case. The plus sign after the $T$ on the righthand side has been chosen in order to describe a collapsing dust cloud.

As has been discussed, for example, in [17], Eq. (64) gives for a Schwarzschild spacetime a relation between Killing time and the time used by families of freely falling observers. Each family is characterized by a fixed value of $E$. All observers within one family start at infinity with the speed $v_{\infty}$, where $E=v_{\infty}^{2} /\left(2\left(1-v_{\infty}^{2}\right)\right)$. In the marginal case they thus start with zero initial speed. In this case Eq. (69) gives the relation between the Schwarzschild time and the Painlevé-Gullstrand time [17].

\section{F. Reconstructing $E$ and $\tau_{0}$ from the canonical data}

The aim of this subsection is to reconstruct the local energy $E$ and the singularity curve $\tau_{0}$ from the canonical data. We have performed this reconstruction already for the mass distribution function, see (36). The three functions $E$, $\tau_{0}$, and $F$ determine the classical LTB model completely. There are several reasons for reconstructing these quantities. First of all, they have a clear physical meaning, whereas this is not so evident for the canonical coordinates. This is the reason why we have replaced the canonical coordinates $L$ and $P_{L}$ with $F$ and $P_{F}$. One might hope that it is possible to turn $E$ or $\tau_{0}$ into canonical coordinates, although this has not been achieved yet. But even without this, they help interpreting the canonical coordinates.

We shall first demonstrate the following connection between the energy densities $\Gamma$ and $P_{\tau}$ :

$$
P_{\tau}=\frac{\Gamma}{2 \sqrt{2 E+1}}
$$

for an arbitrary foliation. Consider the momentum constraint

$$
\begin{aligned}
0 & =\tau^{\prime}+\frac{R^{\prime} \bar{P}_{R}}{P_{\tau}}+\frac{P_{F} \Gamma}{P_{\tau}} \\
& =\tau^{\prime} \pm \frac{R^{\prime}}{\mathcal{F}} \sqrt{\frac{\Gamma^{2}}{4 P_{\tau}^{2}}-\mathcal{F}}+\frac{P_{F} \Gamma}{P_{\tau}},
\end{aligned}
$$

where, in the second step, we have used the Hamiltonian constraint (60) in the form

$$
\bar{P}_{R}= \pm \frac{P_{\tau}}{\mathcal{F}} \sqrt{\frac{\Gamma^{2}}{4 P_{\tau}^{2}}-\mathcal{F}} .
$$

Comparing (72) with (62) we can read off Eq. (70), which is also a constraint. Equation (70) then gives us a simple expression for $E$ in terms of the canonical variables,

$$
1+2 E=\frac{\Gamma^{2}}{4 P_{\tau}^{2}} \stackrel{(60)}{=}-\frac{\Gamma^{2}}{\mathcal{F} P_{R}-\Gamma^{2} /(4 \mathcal{F})} .
$$

Note that, using the solution (6) of Einstein's equation, we have an expression for $\tau_{0}-\tau$, that is, for the remaining proper dust time until the dust shell reaches the singularity,

$$
\tau_{0}-\tau=\frac{R^{3 / 2} Q\left(-\frac{E R}{F}\right)}{\sqrt{F}} .
$$

Since we know now how to express $E$ by canonical coordinates, this equation gives us an expression for the bang time $\tau_{0}$ in terms of canonical coordinates.

Now one can express $\bar{P}_{R}$ in terms of $F$ and $E$. Inserting the expression for $P_{\tau}$ into (73), we have

$$
\begin{aligned}
\bar{P}_{R} & =\frac{1}{\mathcal{F}} \frac{\Gamma}{2 \sqrt{2 E+1}}( \pm 1) \sqrt{2 E+1-\mathcal{F}} \\
& =\frac{1}{\mathcal{F}} \frac{\Gamma}{2 \sqrt{2 E+1}} \partial_{\tau} R .
\end{aligned}
$$

\section{G. Hamiltonian equations of motion}

Here we shall give the Hamilton equations of motion and derive Einstein's equation (2) from them. The Hamiltonian equations are generally given by

$$
\dot{X}=\left\{X, \mathcal{H}[N]+\mathcal{H}_{r}\left[N^{r}\right]\right\},
$$




$$
\dot{P}_{X}=\left\{P_{X}, \mathcal{H}[N]+\mathcal{H}_{r}\left[N^{r}\right]\right\},
$$

where we have introduced the smeared constraints

$$
\begin{gathered}
\mathcal{H}[N]=\int_{0}^{\infty} d r N(r) H(r), \\
\mathcal{H}_{r}\left[N^{r}\right]=\int_{0}^{\infty} d r N^{r}(r) H_{r}(r) .
\end{gathered}
$$

Starting from the action (57), the Hamiltonian equations of motion are ${ }^{1}$

$$
\begin{gathered}
\dot{\tau}=2 N P_{\tau}+N^{r} \tau^{\prime}, \\
\dot{P}_{\tau}=\left(N_{r} P_{\tau}\right)^{\prime}, \\
\dot{R}=2 N \mathcal{F} \bar{P}_{R}+N_{r} R^{\prime}, \\
\dot{\bar{P}}_{R}=-N\left(\frac{F \bar{P}_{R}^{2}}{R^{2}}+\frac{\Gamma^{2} F}{4 \mathcal{F}^{2} R^{2}}\right)+\left(N^{r} \bar{P}_{R}\right)^{\prime}, \\
\dot{\Gamma}=\left(N^{r} \Gamma\right)^{\prime}, \\
\dot{P}_{\Gamma}=N \frac{\Gamma}{2 \mathcal{F}}+N^{r} P_{\Gamma}^{\prime}-\int_{r}^{\infty} d \tilde{r} N(\tilde{r})\left(\frac{(84)}{R(\tilde{r})}+\frac{\Gamma_{R}^{2}(\tilde{r})}{4 \mathcal{F}^{2}(\tilde{r}) R(\tilde{r})}\right) .
\end{gathered}
$$

Consider now

$$
\partial_{\tau} R=\frac{\dot{R}}{\dot{\tau}} \stackrel{N^{r}=0}{=} \frac{N 2 \mathcal{F} \bar{P}_{R}}{N 2 P_{\tau}} \stackrel{(70)}{=} \frac{\mathcal{F} \bar{P}_{R} 2 \sqrt{2 E+1}}{\Gamma},
$$

which we can solve for $\bar{P}_{R}$. Inserting this expression into the Hamiltonian constraint (60) and again using (70) gives

$$
0=H=\frac{\Gamma^{2}}{4(2 E+1)}+\frac{\Gamma^{2}\left(\partial_{\tau} R\right)^{2}}{\mathcal{F} 4(2 E+1)}-\frac{\Gamma^{2}}{4 \mathcal{F}} .
$$

Solving for $\left(\partial_{\tau} R\right)^{2}$ leads to Einstein's equation (2). Note that we did not have to specify the lapse function.

One can show, moreover, that $F, E$, and $\tau_{0}$ are constants of motion, that is, they have vanishing Poisson brackets with the Hamiltonian constraint. In their given local form they do, of course, not commute with the momentum constraint, since the latter generates their transformation with respect to a relabeling $r \rightarrow f(r)$. One would, however, expect that a suitable nonlocal form commutes with $H_{r}$ and thus turns them into real "observables" (similar to what one would expect to happen with the geometric operators in loop quantum gravity [4]). This would coincide with the interpretation of them being the physically relevant variables energy and bang time.

We also note that since (for vanishing shift)

$$
\dot{\tau}=N\left(2 P_{\tau}+F^{\prime}\right),
$$

\footnotetext{
${ }^{1}$ Note that $\delta F(r) / \delta \Gamma(\bar{r})=\theta(r-\bar{r})$.
}

$N$ has no longer the interpretation of being the ratio of proper time to ADM time, cf. [4]. The reason is that we have squared the original version of the Hamiltonian constraint, see Appendix A. If we define a new version of the constraint by taking a square root,

$$
H_{\uparrow}=P_{\tau}-\sqrt{-\mathcal{F} \bar{P}_{R}^{2}+\frac{\Gamma^{2}}{4 G^{2} \mathcal{F}}} \approx 0,
$$

we find that

$$
\left\{\tau, \mathcal{H}_{\uparrow}\left[N^{\dagger}\right]\right\}=N^{\uparrow}
$$

and recover for the lapse function $N^{\uparrow}$ the old interpretation.

We finally remark that the algebra of the constraints cannot be of the general form (given, for example, in [4]), because we have used the momentum constraint to eliminate $P_{F}$ in the Hamiltonian constraint. In fact, a short calculation gives

$$
\{\mathcal{H}[N], \mathcal{H}[M]\}=0,
$$

$$
\begin{gathered}
\left\{\mathcal{H}_{r}\left[N^{r}\right], \mathcal{H}[N]\right\}=\mathcal{H}\left[N_{, r} N^{r}-N^{r} N_{r}^{r}\right], \\
\left\{\mathcal{H}_{r}\left[N^{r}\right], \mathcal{H}_{r}\left[M^{r}\right]\right\}=\mathcal{H}_{r}\left[\left[N^{r}, M^{r}\right]\right] .
\end{gathered}
$$

We note that the Poisson bracket of the Hamiltonian with itself vanishes, Eq. (92), in contrast to the general case where it closes on the momentum constraint. The other brackets coincide with the general case. The transformations generated by the Hamiltonian constraint can thus no longer be interpreted as hypersurface deformations. They are in general not orthogonal to the hypersurfaces, but act along the flow lines of dust.

\section{DIFFEOMORPHISM INVARIANT QUANTUM STATES}

\section{A. Quantum constraints}

We shall now apply the quantization procedure proposed by Dirac and turn the classical constraints into quantum operators, cf. [4]. The starting points are thus the expressions (60) and (61).

The translation of Poisson brackets into commutators is achieved in the Schrödinger representation by substituting

$$
\begin{gathered}
P_{\tau}(r) \rightarrow \frac{\hbar}{i} \frac{\delta}{\delta \tau(r)}, \quad \bar{P}_{R}(r) \rightarrow \frac{\hbar}{i} \frac{\delta}{\delta R(r)}, \\
P_{\Gamma}(r) \rightarrow \frac{\hbar}{i} \frac{\delta}{\delta \Gamma(r)}
\end{gathered}
$$

and acting with them on wave functionals. The Hamiltonian constraint (60) then leads to the WDW equation, 


$$
\begin{aligned}
& {\left[-G \hbar^{2}\left(\frac{\delta^{2}}{\delta \tau(r)^{2}}+\mathcal{F} \frac{\delta^{2}}{\delta R(r)^{2}}+A(R, F) \delta(0) \frac{\delta}{\delta R(r)}\right.\right.} \\
& \left.\left.\quad+B(R, F) \delta(0)^{2}\right)-\frac{\Gamma^{2}}{4 G \mathcal{F}}\right] \Psi\left[\tau\left(r^{\prime}\right), R\left(r^{\prime}\right), \Gamma\left(r^{\prime}\right)\right]=0,
\end{aligned}
$$

where $A$ and $B$ are smooth functions of $R$ and $F$ that encapsulate the factor ordering ambiguities. We have introduced divergent quantities such as $\delta(0)$ in order to indicate that the factor ordering problem is unsolved and can be dealt with only after some suitable regularization has been performed, cf. [18]. That is, one would like to choose the terms proportional to $\delta(0)$ in such a way that the constraint algebra closes, which is usually called "Dirac consistency."

Quantizing the momentum constraint (61) by using (95) gives

$$
\begin{aligned}
{\left[\tau^{\prime} \frac{\delta}{\delta \tau(r)}\right.} & \left.+R^{\prime} \frac{\delta}{\delta R(r)}-\Gamma\left(\frac{\delta}{\delta \Gamma(r)}\right)^{\prime}\right] \\
& \times \Psi\left[\tau\left(r^{\prime}\right), R\left(r^{\prime}\right), \Gamma\left(r^{\prime}\right)\right]=0 .
\end{aligned}
$$

Up to now, the quantum constraint equations have been formulated only in a formal way. The next subsection is devoted to the application of a lattice regularization.

\section{B. Lattice regularization}

We follow here the suggestion made in [12] and consider a one-dimensional lattice given by a discrete set of points $r_{i}$ separated by a distance $\sigma$. In [12], the distance was allowed to depend on the point $r_{i}$. We restrict here to a constant size $\sigma$ because this choice is sufficient to perform the continuum limit and is, in addition, more transparent. In order that the momentum constraint is fulfilled in the continuum limit, it is important to start with a corresponding ansatz for the wave functional before putting it on the lattice. We therefore make the ansatz

$$
\Psi[\tau(r), R(r), \Gamma(r)]=U\left(\int d r \Gamma(r) \mathcal{W}(\tau(r), R(r), \Gamma(r))\right),
$$

where $U: \mathbb{R} \rightarrow \mathbb{C}$ is at this stage some arbitrary (differentiable) function. Using $\Gamma$ in the exponent instead of $R^{\prime}$ or $\tau^{\prime}$ is suggested by the form of the WDW equation (absence of derivatives with respect to $\Gamma$ ) and the fact that $F^{\prime}=\Gamma$ is related to the energy density. The ansatz has to be compatible with the lattice, which means that it has to factorize into different functions for each lattice point. So we have to make the choice $U=\exp$, which gives

$$
\begin{aligned}
& \Psi[\tau(r), R(r), \Gamma(r)] \\
& \quad=\exp \left(\int d r \Gamma(r) \mathcal{W}(\tau(r), R(r), F(r))\right)
\end{aligned}
$$

$$
\begin{aligned}
& =\exp \left(\lim _{\sigma \rightarrow 0} \sum_{i} \sigma \Gamma_{i} \mathcal{W}_{i}\left(\tau\left(r_{i}\right), R\left(r_{i}\right), F\left(r_{i}\right)\right)\right) \\
& =\lim _{\sigma \rightarrow 0} \prod_{i} \exp \left(\sigma \Gamma_{i} \mathcal{W}_{i}\left(\tau\left(r_{i}\right), R\left(r_{i}\right), F\left(r_{i}\right)\right)\right) \\
& =\lim _{\sigma \rightarrow 0} \prod_{i} \Psi_{i}\left(\tau\left(r_{i}\right), R\left(r_{i}\right), \Gamma\left(r_{i}\right), F\left(r_{i}\right)\right),
\end{aligned}
$$

where

$$
F\left(r_{i}\right)=\sum_{j=0}^{i} \sigma \Gamma_{j}
$$

As in [12] we implement the formal expression $\delta(0)$ onto the lattice as follows:

$$
\delta(0) \rightarrow \lim _{\sigma \rightarrow 0} \frac{1}{\sigma} .
$$

The lattice version of the WDW equation (96) then reads

$$
\begin{array}{r}
{\left[G \hbar^{2}\left(\frac{\partial^{2}}{\partial \tau_{j}^{2}}+\mathcal{F}_{j} \frac{\partial^{2}}{\partial R_{j}^{2}}+A\left(R_{j}, F_{j}\right) \frac{\partial}{\partial R_{j}}\right)\right.} \\
\left.+B(R, F)+\frac{\sigma^{2} \Gamma}{4 G \mathcal{F}_{j}}\right] \Psi_{j}=0 .
\end{array}
$$

We now insert the ansatz (102) and make for convenience the redefinition $\mathcal{W}=i W / 2$. This leads to

$$
\begin{array}{r}
\frac{\sigma^{2} \Gamma_{i}^{2}}{4}\left[G \hbar^{2}\left(\frac{\partial W(\tau, R, F)}{\partial \tau}\right)^{2}+G \hbar^{2} \mathcal{F}\left(\frac{\partial W(\tau, R, F)}{\partial R}\right)^{2}-\frac{1}{G \mathcal{F}}\right] \\
+\frac{\sigma \Gamma_{i}}{2}\left[G \hbar^{2}\left(\frac{\partial^{2}}{\partial \tau^{2}}+\mathcal{F} \frac{\partial}{\partial R^{2}}+A(R, F) \frac{\partial}{\partial R}\right) W(\tau, R, F)\right] \\
+B(R, F)=0 .
\end{array}
$$

In order for this to be fulfilled independent of the choice of $\sigma$ (and thus also in the limit $\sigma \rightarrow 0$ ) one is led to the following three equations,

$$
\left(G \hbar \frac{\partial W(\tau, R, \Gamma)}{\partial \tau}\right)^{2}+\mathcal{F}\left(G \hbar \frac{\partial W(\tau, R, \Gamma)}{\partial R}\right)^{2}-\frac{1}{\mathcal{F}}=0,
$$

$$
\left(\frac{\partial^{2}}{\partial \tau^{2}}+\mathcal{F} \frac{\partial^{2}}{\partial R^{2}}+A(R, \Gamma) \frac{\partial}{\partial R}\right) W(\tau, R, \Gamma)=0,
$$

and

$$
B(R, \Gamma)=0 .
$$

The first equation, (108), the Hamilton-Jacobi equation, is the same as in [9], Eq. (5.11) and in [11], Eq. (12). The second equation presents an additional restriction on solutions of (108). The last equation (110) tells us that working on the lattice is only possible if the factor ordering does not contribute to the potential term. If we find solutions to all three equations, we can do all other calculations on the 
lattice, since these solutions have a well-defined continuum limit and satisfy the momentum constraint.

At this point we have to make a few comments on the regularization procedure we are using. It was already noted that the lattice regularization does not solve the factor ordering problem. The lattice regularization just represents an $a d$ hoc regularization in which the divergent terms have to cancel each other. Put differently, it is equivalent to a DeWitt type of regularization [which means setting $\delta(0)=$ 0 ] with an additional constraint on the solutions. It has to be noted that the Hamilton-Jacobi equation, Eq. (108), can equivalently be obtained from the highest order of a WKB approximation [11].

We have already emphasized that the signature in the kinetic part of the Hamiltonian constraint (60) can change from elliptic (outside the horizon) to hyperbolic (inside the horizon). This thus occurs for the kinetic term of the WDW equation (96), too. As already noted above, a similar phenomenon can be found in the context of quantum cosmology [16]. As discussed in [19], we can say that the part inside the horizon is always classically allowed, whereas this is not necessarily the case for the outside part. The usual initial value problem appropriate for hyperbolic equations can thus only be applied for the region corresponding to the black-hole interior.

Since we have found from our special ansatz the two equations (108) and (109), we cannot expect for them a well-posed initial value problem to hold because the system is in general overdetermined. The goal pursued in this paper is not the discussion of boundary value problems but to find a class of exact solutions to all quantum constraints and to draw from them physical conclusions.

It is instructive to check that one can get Einstein's equation (2) from the Hamilton-Jacobi equation (108). A class of solutions is given by the complete integral

$$
G \hbar W(\tau, R, a, b)=b+a \tau \pm \int d R \frac{\sqrt{1-a^{2}} \overline{\mathcal{F}}}{\mathcal{F}},
$$

which is identical to the expression occurring in (64), cf. also Eq. (3.3) in [17]. Since we made an ansatz of the form $\Psi \equiv \exp (i S / G \hbar) \equiv \exp \left(i \int\left(F^{\prime} / 2\right) W\right)$, the relation

$$
\begin{aligned}
P_{R} & =\frac{F^{\prime}}{2} \frac{\partial}{\partial R} W \\
& =\frac{F^{\prime}}{2} \frac{\sqrt{1-a^{2} \mathcal{F}}}{\mathcal{F}}
\end{aligned}
$$

should hold. Using the Hamiltonian equation of motion for zero shift we have

$$
\dot{R}=2 N \mathcal{F} P_{R}
$$

Inserting this in (113) gives

$$
\left(\frac{\dot{R}}{N F^{\prime}}\right)^{2}=1-a^{2}+a^{2} \frac{F}{R} \text {. }
$$

This equation should be equivalent to Einstein's equation given by

$$
\left(\frac{d R}{d \tau}\right)^{2}=\frac{F}{R}+2 E .
$$

For $a=0$ the right-hand side of (115) is equal to 1 . This is a limiting case. As discussed in [17], it corresponds to observers that start at $\mathcal{J}^{-}$using the EddingtonFinkelstein coordinate $v$ as their time coordinate. For an arbitrary $a \neq 0$ we can write

$$
\left(\frac{\dot{R}}{N F^{\prime} a}\right)^{2}=\frac{1}{a^{2}}-1+\frac{F}{R}
$$

and can thus identify $2 E=1 / a^{2}-1$. Hence, $a$ has the same interpretation as in (64). Letting $a$ go to zero implies that $E$ diverges.

\section{Solutions}

We have obtained above the two equations (108) and (109), which have to be satisfied in order to get a diffeomorphism invariant solution to the WDW equation. (We set now $G=1=\hbar$.) Looking for particular solutions of the separating form $W=\alpha(\tau)+\beta(R)$, we recognize immediately that this system of equations is consistent only for special factor orderings. The particular factor ordering $A(R, F)=F /\left(2 R^{2}\right)$ used in $[11,12]$ is not among them. (For the WKB solutions used in [11] this is irrelevant.)

Tackling the problem from the opposite point of view, one can ask for which factor orderings we $d o$ get a separating solution. We find

$$
A(R, F)=-\frac{F}{2 R^{2}}\left(1+\frac{1}{1-a^{2} \mathcal{F}}\right)
$$

This leads to

$$
W(\tau, \Gamma, R, a)=\text { const } \pm a \tau \pm \int d R \frac{\sqrt{1-a^{2}} \overline{\mathcal{F}}}{\mathcal{F}} .
$$

These are identical to the solutions that were obtained in the solution of the Hamilton-Jacobi equation, cf. (66) and (111). Thus we can again identify $2 E=1 / a^{2}-1$. Since classically $E \geq-1 / 2$, it follows that $a$ should be real. Using (70), we can show that this is consistent:

$$
\hat{P}_{\tau} \Psi_{a}= \pm \frac{a \Gamma}{2} \Psi_{a}= \pm \frac{\Gamma}{2 \sqrt{2 E+1}} \Psi_{a}
$$

where we have defined

$$
\Psi_{a}[\tau, R, \Gamma]=\exp \left(\frac{i}{2} \int_{0}^{\infty} d r \Gamma W(\tau, R, F, a)\right) .
$$

The integral appearing in (119) has been evaluated above for the various cases, see (66)-(68). We recognize, in particular, from (68) that the wave function becomes a real exponential in the region $R<F a^{2} /\left(a^{2}-1\right)$. As can be seen from (24), this is the region that is classically 
forbidden; the real, nonoscillatory, behavior of the wave function is thus no surprise.

Surprisingly, Eq. (119) gives in fact already the complete class of solutions. This is shown in Appendix B. There thus exist no nonseparating solutions, given the ansatz made where the full wave functional factorizes into functions on the respective lattice points. But we emphasize that we have succeeded in finding exact solutions to all quantum constraints. Other solutions to the full WDW equation and momentum constraints would necessarily couple the infinitely many shells comprising the dust cloud; to find them would demand a regularization scheme that is much beyond the scope of this paper. In a sense, the factor ordering chosen here leads to quantum states for which the WKB form is "exact," cf. the analogous situation with the models discussed in $[19,20]$.

For definiteness we will consider in the following only the positive sign in front of $a \tau$ in (119). For $a=0$, which corresponds to $E=\infty$, the solutions are particularly simple. They read

$$
\begin{gathered}
W_{\text {in }}^{ \pm}(\tau, \Gamma, R, a=0)=\mathrm{const} \pm(R+F \ln (F-R)), \\
W_{\text {out }}^{ \pm}(\tau, \Gamma, R, a=0)=\mathrm{const} \pm(R+F \ln (R-F)),
\end{gathered}
$$

where "in" refers to the region inside the horizon and "out" to the region outside the horizon. For $a=1$, which corresponds to the marginal model $E=0$, we obtain the solutions

$W_{\mathrm{in}}^{ \pm}(\tau, \Gamma, R, a=1)=\mathrm{const}+\tau$

$$
\pm 2 \sqrt{F}\left[\sqrt{R}-\sqrt{F} \tanh ^{-1}\left(\sqrt{\frac{R}{F}}\right)\right],
$$

$W_{\text {out }}^{ \pm}(\tau, \Gamma, R, a=1)=$ const $+\tau$

$$
\pm 2 \sqrt{F}\left[\sqrt{R}-\sqrt{F} \tanh ^{-1}\left(\sqrt{\frac{F}{R}}\right)\right] .
$$

These solutions were already found in [9] and discussed further in [11].

The full solutions for the marginal model read

$$
\begin{aligned}
& \Psi_{\text {in/out }}^{ \pm}[\tau, R, \Gamma] \\
& \quad=\Psi_{\Gamma \text { in } / \text { out }}[\Gamma] \exp \left(\frac{i}{2} \int_{0}^{\infty} d r \Gamma(r) W_{\text {in } / \text { out }}^{ \pm}(\tau, R, \Gamma, a=1)\right) .
\end{aligned}
$$

The solutions $\Psi_{\text {in }}^{ \pm}$and $\Psi_{\text {out }}^{ \pm}$have to be matched at the horizon, that is, both the states and their $R$ derivatives should agree there. As can be seen from (124) and (125), however, the phases of the states diverge there. One thus has to perform an analytic continuation: We write $R-F=$ $\epsilon \exp (i \varphi), \epsilon>0$, and compare the states at $\varphi=\pi / 2$, that is, at $R=R_{h} \equiv F+i \epsilon$. One finds from this comparison that the states are related as

$$
\begin{aligned}
\Psi_{\text {in }}^{ \pm} & {\left[\tau, R_{h}, \Gamma\right] } \\
& =\frac{\Psi_{\Gamma \text { in }}[\Gamma]}{\Psi_{\Gamma \text { out }}[\Gamma]} \exp \left(\mp \frac{\pi}{2} \int_{0}^{\infty} d r \Gamma(r) F(r)\right) \Psi_{\text {out }}^{ \pm}\left[\tau, R_{h}, \Gamma\right] .
\end{aligned}
$$

The states $\Psi_{\text {in }}^{ \pm}$and $\Psi_{\text {out }}^{ \pm}$can be set equal at the horizon if we exploit the freedom to choose $\Psi_{\Gamma}[\Gamma]$. One thus gets a relation between $\Psi_{\Gamma \text { in }}^{ \pm}[\Gamma]$ and $\Psi_{\Gamma \text { out }}^{ \pm}[\Gamma]$,

$$
\begin{aligned}
\Psi_{\Gamma \text { in }}^{ \pm}[\Gamma] & =\exp \left(\mp \frac{\pi}{2} \int_{0}^{\infty} d r \Gamma(r) F(r)\right) \Psi_{\Gamma \text { out }}^{ \pm}[\Gamma] \\
& =\exp \left(\mp \frac{\pi}{4}\left(F^{2}(\infty)-F^{2}(0)\right)\right) \Psi_{\Gamma_{\text {out }}^{ \pm}}^{ \pm}[\Gamma] \\
& =\exp \left(\mp \pi M^{2}\right) \Psi_{\Gamma \text { out }}^{ \pm}[\Gamma] .
\end{aligned}
$$

On the lattice this reads

$$
\Psi_{\text {Гin }}^{ \pm}\left(\Gamma_{i}, F_{i}\right)=\exp \left(\mp \pi \omega_{i} F_{i}\right) \Psi_{\text {Гout }}^{ \pm}\left(\Gamma_{i}, F_{i}\right) .
$$

One can easily check that with this choice the derivatives of the states at the horizon coincide as well. We also remark that the alternative choice $R=F-i \epsilon$ would lead to a switch of sign in the exponent of (130).

We mention that the factor acquired when crossing the horizon might be connected to the entropy of the black hole [19]. In fact, we recognize that the absolute value of the exponent is one quarter of the Bekenstein-Hawking entropy.

The solutions (119) are all solutions that can occur on the lattice for the factor ordering (118). Here we want to extend these solutions to the continuum. The solutions on the lattice contain two free parameters, $a_{i}$ and $b_{i}$,

$$
\Psi_{i}=e^{b_{i} \sigma \Gamma_{i} F_{i}} e^{(i / 2) \sigma \Gamma\left(a_{i} \tau_{i}+\int d R\left[\left(\sqrt{1-a_{i}} \overline{\mathcal{F}}_{i}\right) / \mathcal{F}_{i}\right]\right)} .
$$

(We have for simplicity considered only one sign in the exponent.) We know that $a_{i}$ is connected with the local energy $E$ via $2 E_{i}+1=1 / a_{i}^{2}$. In general we have $E=$ $E(r)$, and thus it would be natural to demand that $a=a(r)$. But with this explicit dependence on $r$ the momentum constraint would not be satisfied. The only possible way out of this is to use an implicit dependence $a(F(r))$ in order to get solutions fulfilling the momentum constraint. The expression 


$$
\begin{aligned}
\Psi[\tau, R, \Gamma]= & e^{\int d r b(F(r)) \Gamma} \exp \left\{\frac{i}{2} \int d r \Gamma[a(F(r)) \tau\right. \\
& \left.\left.+\int d R \frac{\sqrt{1-a(F(r)) \mathcal{F}}}{\mathcal{F}}\right]\right\}
\end{aligned}
$$

is still a solution, since the Hamiltonian constraint does not contain a derivative with respect to $\Gamma$ or $F$. Hence we arrive at a family of solutions containing two arbitrary functions $a(x)$ and $b(x)$. It is clear that $a(x)$ is connected with $E$ via $2 E(r)+1=1 / a^{2}(F(r))$. One might in principle wish to construct wave packets by superposing wave functions with different $a$, that is, with different energies. However, since the factor ordering depends on $E$, this does not seem feasible.

\section{DISCUSSION}

Let us first summarize the main results of our paper. As for the classical part, we have extended the canonical formalism to the nonmarginal case. We have shown, in particular, that (60) holds for all values of $E$. We have succeeded in expressing the observables $F, E$, and $\tau_{0}$, which determine the classical model completely, in terms of the canonical variables. We have presented a new and improved method to cope with the boundary action in the canonical formalism. This renders the formalism more transparent. We have presented the general relation between dust proper time and Killing time and noted the similarity of dust time with the generalized PainlevéGullstrand time discussed in [17].

As for the quantum part, we have presented a lattice regularization that correctly implements the momentum constraint in the continuum limit. For a particular factor ordering (that, however, depends on $E$ ) we have succeeded to give exact solutions to all constraints. We have shown that, given the general ansatz for the wave functional on the lattice, these are the only solutions. This means that these are the only solutions for which the states describing the dust cloud factorize into infinitely many states corresponding to the various shells forming the cloud. We have also extended these solutions into the continuum.

The discussion of the quantum LTB model is far from being exhausted. We thus want to conclude with an outlook on future work. First, we have mentioned in Sec. III C a possible connection between the exponential factor in (130) and the Bekenstein-Hawking entropy. Perhaps it will be possible to recover this entropy as an entanglement entropy from these quantum states. This could provide a first step towards its general understanding. Second, it would be of great importance to study the possible singularity avoidance of the quantum LTB model. This would include also an understanding of the role of the naked singularities in the classical model. We recall that singularity avoidance was a main feature of the quantization of dust shells [3]. Singularity avoidance was also shown in various models of loop quantum gravity [5]. A third issue would thus be to develop the loop quantization of the LTB model and compare its results with the results obtained from the WDW equation.

Extending the discussion of Hawking radiation in $[11,12]$, we plan to derive the corresponding Bogoliubov coefficients from the exact quantum states found in this paper. This should yield in the appropriate limits the thermal Hawking spectrum plus quantum gravitational correction terms. This is currently under investigation.

\section{ACKNOWLEDGMENTS}

We thank T.P. Singh, Carsten Weber, L.C.R. Wijewardhana, and Louis Witten for discussions. C. K. is grateful to the Max-Planck-Institut für Gravitationsphysik in Golm, Germany, for its kind hospitality while part of this work was done.

\section{APPENDIX A: SIMPLIFICATION OF THE HAMILTONIAN CONSTRAINT}

Here we show how it is possible to eliminate $P_{F}$ from (58) by using (59) and to obtain the new, relatively simple, version (60) of the Hamiltonian constraint.

The momentum constraint (59) can be solved for $\bar{P}_{R}$, which gives

$$
\bar{P}_{R}=-\frac{\tau^{\prime} P_{\tau}-\Gamma P_{\Gamma}^{\prime}}{R^{\prime}} .
$$

Inserting this expression into the Hamiltonian constraint (58) and noting that $L^{2}=R^{\prime 2} / \mathcal{F}-4 \mathcal{F} P_{\Gamma}^{\prime 2}$ gives

$$
\begin{aligned}
2 P_{\tau} \sqrt{L^{2}+\tau^{\prime 2}} & =\left[\frac{\Gamma R^{\prime}}{\mathcal{F}}+\frac{4 \mathcal{F} P_{\Gamma}^{\prime}}{R^{\prime}}\left(\tau^{\prime} P_{\tau}-\Gamma P_{\Gamma}^{\prime}\right)\right] \\
& =\Gamma L^{2}+4 \mathcal{F} \tau^{\prime} P_{\Gamma}^{\prime} P_{\tau} .
\end{aligned}
$$

Squaring the resulting equation yields

$$
\begin{aligned}
4 R^{\prime 2} P_{\tau}^{2} L^{2}+4 R^{\prime 2} P_{\tau}^{2} \tau^{\prime 2}= & \Gamma^{2} L^{4}+8 \mathcal{F} L^{2} \Gamma \tau^{\prime} P_{\Gamma}^{\prime} P_{\tau} \\
& +\underbrace{16 \mathcal{F}^{2} \tau^{\prime 2} P_{\Gamma}^{2} P_{\tau}^{2} .}_{4 \tau^{2} P_{\tau}^{2}\left(R^{\prime}-\mathcal{F} L^{2}\right)}
\end{aligned}
$$

The second term on the left cancels with the corresponding term on the right. After dividing by $L^{2}$ one has

$$
4 R^{\prime 2} P_{\tau}^{2}+4 \mathcal{F} P_{\tau}^{2} \tau^{\prime 2}=\Gamma^{2} \underbrace{R^{\prime 2} / \mathcal{F}-4 \mathcal{F} P_{\Gamma}^{2}}_{L^{2}}+8 \mathcal{F} \Gamma \tau^{\prime} P_{\Gamma}^{\prime} P_{\tau} \text {. }
$$

If we combine the second term on the left-hand side with the second and third terms on the right-hand side, it is possible to use the momentum constraint to eliminate $P_{\Gamma}^{\prime}$,

$$
4 R^{\prime 2} P_{\tau}^{2}=\frac{\Gamma^{2} R^{\prime 2}}{\mathcal{F}}-4 \mathcal{F} \underbrace{\left(\tau^{\prime} P_{\tau}-\Gamma P_{\Gamma}^{\prime}\right)^{2}}_{R^{\prime 2} \bar{P}_{R}^{2}} .
$$


This then yields the desired expression for (60):

$$
P_{\tau}^{2}+\mathcal{F} \bar{P}_{R}^{2}-\frac{\Gamma^{2}}{4 \mathcal{F}}=0
$$

\section{APPENDIX B: UNIQUENESS OF QUANTUM SOLUTIONS}

Here we shall demonstrate that the separating solutions found from (108) and (109) are unique.

Equation (108) may be solved by the ansatz

$$
\partial_{\tau} W=\frac{\cos \eta}{\sqrt{\mathcal{F}}}, \quad \partial_{R} W=\frac{\sin \eta}{\mathcal{F}} .
$$

The function $\eta$ has to fulfill the integrability condition

$$
-\frac{\sin \eta}{\sqrt{\mathcal{F}}} \partial_{R} \eta-\frac{\cos \eta}{2 \mathcal{F}^{3 / 2}} \partial_{R} \mathcal{F}=\frac{\cos \eta}{\mathcal{F}} \partial_{\tau} \eta,
$$

which leads to

$$
\partial_{\tau} \eta=-\sqrt{\mathcal{F}} \tan \eta \partial_{R} \eta-\partial_{R} \sqrt{\mathcal{F}} .
$$

Inserting ansatz (B1) into (109) gives another equation for $\eta$

$$
-\frac{1}{\sqrt{\mathcal{F}}} \partial_{\tau} \eta+\cot \eta \partial_{R} \eta-\partial_{R} \ln \mathcal{F}+\frac{A}{\mathcal{F}}=0 .
$$

So the ansatz $A=\mathcal{F} \partial_{R} \ln (\mu \mathcal{F})$ yields

$$
\partial_{\tau} \eta=\sqrt{\mathcal{F}} \cot \eta \partial_{R} \eta+\sqrt{\mathcal{F}} \partial_{R} \ln \mu .
$$

The integrability conditions (B3) and (B5) have to be consistent. This gives

$$
-\sqrt{\mathcal{F}} \tan \eta \partial_{R} \eta-\partial_{R} \sqrt{\mathcal{F}}=\sqrt{\mathcal{F}} \cot \eta \partial_{R} \eta+\sqrt{\mathcal{F}} \partial_{R} \ln \mu .
$$

By an elementary manipulation we obtain

$$
\partial_{R} \ln (\sqrt{\mathcal{F}} \mu \tan \eta)=0,
$$

and thus

$$
\begin{gathered}
\tan \eta=\frac{\alpha(\tau)}{\mu \sqrt{\mathcal{F}}}, \quad \sin \eta=\frac{\alpha(\tau)}{\sqrt{\alpha^{2}+\mu^{2} \mathcal{F}}}, \\
\cos \eta=\frac{\mu \sqrt{\mathcal{F}}}{\sqrt{\alpha^{2}+\mu^{2} \mathcal{F}}} .
\end{gathered}
$$

These equations can be used to give expressions for $\partial_{R} \eta$ and $\partial_{\tau} \eta$

$$
\begin{gathered}
\partial_{R} \eta=\frac{\alpha \mu^{2} \mathcal{F}}{\alpha^{2}+\mu^{2} \mathcal{F}} \partial_{R}\left(\frac{1}{\mu \mathcal{F}}\right), \\
\partial_{\tau} \eta=\frac{\mu \sqrt{\mathcal{F}}}{\alpha^{2}+\mu^{2} \mathcal{F}} \partial_{\tau} \alpha .
\end{gathered}
$$

Reinserting (B9) and (B10) into (B3) or (B5) leads to

$$
\partial_{\tau} \alpha=\frac{\alpha^{2}}{\mu^{2}} \partial_{R} \mu-\frac{\mu}{2} \partial_{R} \mathcal{F} .
$$

Since $\alpha$ is a function only of $\tau$, and $\mu$ and $\mathcal{F}$ are functions only of $R$, the above equation requires $\alpha=$ const. Hence we have

$$
\frac{\alpha^{2}}{\mu^{3}} \partial_{R} \mu=\frac{1}{2} \partial_{R} \mathcal{F}
$$

which yields

$$
\mu=\frac{\beta}{\sqrt{1-a^{2} \overline{\mathcal{F}}}},
$$

where $\beta$ and $a=\beta / \alpha$ are constants. Then the unique solutions of (108) and (109) are

$$
W=a \tau+\int d R \frac{\sqrt{1-a^{2} \mathcal{F}}}{\mathcal{F}},
$$

which is just (118).
[1] G. Lemaître, Ann. de la Société Scientifique de Bruxelles A 53, 51 (1933); for an English translation see Gen. Relativ. Gravit. 29, 641 (1997).

[2] A. Krasiński, Inhomogeneous Cosmological Models (Cambridge University Press, Cambridge, 1997).

[3] P. Hájíček, in Quantum Gravity: From Theory to Experimental Search, edited by D. Giulini, C. Kiefer, and C. Lämmerzahl (Springer, Berlin, 2003).

[4] C. Kiefer, Quantum Gravity (Clarendon Press, Oxford, 2004).

[5] V. Husain and O. Winkler, Classical Quantum Gravity 22, L127 (2005); M. Bojowald, Phys. Rev. Lett. 95, 061301 (2005); A. Ashtekar and M. Bojowald, Classical Quantum
Gravity 23, 391 (2006); L. Modesto, Phys. Rev. D 70, 124009 (2004).

[6] H. A. Kastrup and T. Thiemann, Nucl. Phys. B425, 665 (1994).

[7] K. V. Kuchař, Phys. Rev. D 50, 3961 (1994).

[8] C. Vaz and L. Witten, Phys. Rev. D 60, 024009 (1999).

[9] C. Vaz, L. Witten, and T. P. Singh, Phys. Rev. D 63, 104020 (2001).

[10] C. Vaz and L. Witten, Phys. Rev. D 63, 024008 (2001); 64, 084005 (2001).

[11] C. Vaz, C. Kiefer, T. P. Singh, and L. Witten, Phys. Rev. D 67, 024014 (2003). 
[12] C. Vaz, L. Witten, and T.P. Singh, Phys. Rev. D 69, 104029 (2004).

[13] K. Kuchař and C. Torre, Phys. Rev. D 43, 419 (1991); J. Brown and K. Kuchař, ibid. 51, 5600 (1995).

[14] J. D. Romano, gr-qc/9501015.

[15] H.D. Zeh, The Physical Basis of the Direction of Time (Springer, Berlin, 2001), 4th ed.
[16] C. Kiefer, Phys. Lett. B 225, 227 (1989).

[17] K. Martel and E. Poisson, Am. J. Phys. 69, 476 (2001).

[18] N. C. Tsamis and R. P. Woodard, Phys. Rev. D 36, 3641 (1987).

[19] T. Brotz and C. Kiefer, Phys. Rev. D 55, 2186 (1997).

[20] D. Louis-Martinez, J. Gegenberg, and G. Kunstatter, Phys. Lett. B 321, 193 (1994). 